\title{
ZUR GESCHICHTE
}

DER

\section{ZELLTHEORIEN.}

EIN VORTRAG

VON

RICHARD ALTMANN.

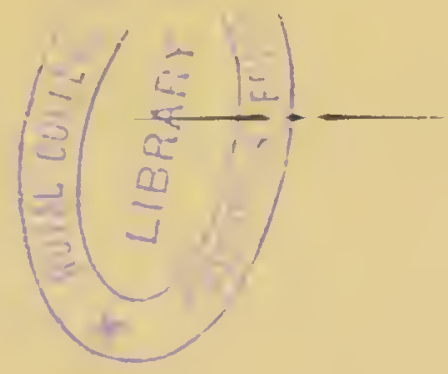

LEIPZIG,

VERLAG VON AMIBR. ABEL

I\$S9. 
Digitized by the Internet Archive in 2015 
Scitdem von Dujardin dic contraktile Substanz oder Sarkode cntcleckt war, hat cliesclbe in Bezug auf die Doutung ihres Wesens und ihrer Verbreitung gar mannigfache Wandlungen erfahren. Dujardin selbst nahm an, dass sic den niederen Thieren zukomme und den Körper derselben im Wesentlichen bilde.

Bald darauf, es sind jetzt gerade 50 Jahre her, fanden Schlciden und Schwann, dass sich der Körper aller Pflanzen und Thiere aus kleinen Territorien aufbaue, wclche Zellen genannt wurden; die Substanz der Zellen selbst aber wurdc in ihren wesentlichen Eigenschaften bald als iibcreinstimmend in allen Organismen erkannt und für dieselbe der Ausdruck Protoplasma gefunden.

Was ist Protoplasma: Hugo von Mohl, welcher diesen Ausdruck aufbrachte, definirt dasselbe als eine rälfflüssige, mit Körnchen gemengtc Substanz; die Körnchen können auch fehlen und es bleibt dann eine glcichförmigc, durchscheinende Massc übrig. ${ }^{x}$

Diese Definition des l'rotoplasmas hat ihre Geltung in Wesentlichen bis auf den heutigen Tag behalten. So bezeichnete Max Schultz $\mathrm{e}^{2}$ ) dasselbe als zähflüssig, zerlegbar in eine glasartig durchsichtige Grundsubstan\% und clie zahl-

I) II. v. Mohl, Ueber die Saftbewegung im Innern der Zellen. Bota. nische Zeitung 1846. S. $7+11.90$.

2) Max śchnltze, Leber Muskelkürperchen und das was man eine Zelle zu nennen habe. Archiv fur Anatomie und l'hysiologie 1861. S. 9. 
reich eingebetteten Körnchen; die letzteren können auch fehlen und die homogene Grundsubstanz übrig lassen. Brücker), indem er den theoretischen Begriff der Zelle abgrenzen will und weder Kern noch Membran als nothwendige Bestandtheile derselben anerkennt, hält für die einfachste Form der Zelle ein Klümpchen Protoplasma, welches wohl eine molekulare Organisation oder Structur besitzt, morphologisch aber nicht zerlegt worden und vielleicht uiberhaupt nicht zerlegbar ist.

Diesen Anschauungen von der Structurlosigkeit des Protoplasmas sind fast alle späteren Autoren, wie Kühne, Lieberkühn und Andere gefolgt, ja dieselben gingen hier insofern zum Theil noch weiter, als sie die lebendige Natur der Körnchen, welche, wenn nicht immer, so doch meist dem Protoplasma sichtbarlich beigemischt sind, mehr weniger bestimmt in Abrede stellen. So erklärt Stricker, dass man nicht berechtigt ist, die Körnchen überhaupt als wesentliche Bestandtheile des Protoplasmas zu betrachten; von den neueren Botanikern, welche sich eingehender mit dem Protoplasma beschäftigt haben, meint Berthold ${ }^{2}$ ), die Körnchen, oder wie sie Hanstein ${ }^{3}$ ) nennt, die Mikrosomen, mögen in vielen Fällen krystallinische oder amorphe feste Ausscheidungen organischer oder unorganischer Natur sein, in andern wieder tröpfchenförmige Ausscheidungen unbekannter Gemische, und Schwarz ${ }^{4}$ ) erklärt von den Körnchen, dass, soweit sie nicht Gerinnungsprodukte der Reagentien sind, es sich bei ihnen um eine Einlagerung unlöslicher körniger Substanzen in das zähflüssige Cytoplasma handelt, welche nur eine metaplasmatische Natur haben. Nur wenige Botaniker haben iberhaupt die Möglichkeit einer feineren

1) Ernst Brücke, Die Elementarorganismen. Wiener Sitzungs" berichte $\mathrm{I} 86 \mathrm{I}$.

2) G. Berthold, Studien iib. Protoplasmamechanik. Leipz. I 886. S. 6 I.

3) T. v. Hanstein: Das Protoplasma. Heidelberg r880. S. 22.

4) F. Schwarz, l)ie morphologische und chcmische \%usammensetzung des Protoplasmas. Breslau 188\%. S. I 37 u. I 38. 
Structur im Cytoplasma erwälnnt; so heisst es in Bezug hierauf in einer der neuesten und objektivsten Erörterungen ${ }^{\mathrm{x}}$ ):

„hn jeder beliebigen lebenden Pflanzenzelle, in der das Cytoplasma eine gewisse Mächtigkeit besitzt, beobachtet man an demselben eine gewisse, ins gräuliche spielende Trübung, die dasselbe granulirt erscheinen lässt. Bei der Kleinheit der in Frage kommenden Gebilde muss es jedoch zur Zeit noch zweifellaft bleiben, ob wir es im Cytoplasma wirklich mit Körnchen von abweichender Lichtbrechung zu thun haben, oder ob die Trubung desselben nicht, wie Naegeli annimmt, mindestens zum grössten Theil dadurch hervorgebracht wird, dass die gesammte Masse des Cytoplasmas von einer grossen Menge winziger Wasser oder Zellsaft enthaltender Vacuolen erfuillt ist."

„Durchmustert man in bezug hierauf die botanische Litteratur, so wird man finden, dass die in dieser Richtung angestellten Beobachtungen noch gänzlich unzureichend sind. und dass ein sicheres Urtheil iiber die feinere Structur des Cytoplasmas zur Zeit noch nicht gefällt werden kann."

„Es soll jedoch mit obigen Worten keineswegs die Möglichkeit einer feineren Structur im Cytoplasma in Abrede gestellt werden; es schien mir nur geboten, darauf hinzuweisen, dass zur Zeit keine mit der nöthigen Kritik angestellten umfassenden Untersuchungen über diesen Gegenstand vorliegen, und dass es jetzt noch nicht möglich ist, in dieser Hinsicht ein irgendwie abschliessendes Urtheil zu fällen."

So konnte Kölliker, indem er in der neuen Ausgabe seines Handbuches der Gewebelehre (1889) in dieser Frage weniger als Autor denn als Referent aufzutreten beniilit ist, die herrschenden Anschauungen der Botaniker sowolll wie der Zootomen dahin zusammenfassen, dass das Protoplasma (S. I I) eine gleichartige, weiche, zähflüssige Substanz sei, in welcher meistens Körnchen und andere Ein-

I) A. Zimmermann, Die Morphologie und Physiologie der Pflnzenzelle. In sichenk's I.ehrbuch der Botanik 1887. S. Io. I2. I3. 
schliusse eingestreut sind; in derselben können im Laufe dei Entwickelung Vacuolen in verschiedenen Grössen und in verschiedenen Mengen auftreten (S. I2); sind dieselben liein, so erscheint das Protoplasma schaumig wie spongiös, werden dieselben grösser. so bildet das Protoplasma Netze, in dessen Maschen sich Flïssigkeit, oder Fetttropfen, Schleimkugeln, Eiweisskörner etc. finden; indem Kölliker eine eigentlich primäre Netzstructur des Protoplasmas, wie sie von Anderen behauptet ist, nicht anzuerkennen scheint, erklärt er Fasern und Fibrillenbildungen als wichtige Einzelheiten des protoplasmatischen Baues (S. I3).

Nach diesen herrschenden Anschauungen hat also das I'rotoplasma seine morphologische Individualisirung in der Form der Zelle gefunden. Die Zelle ist demnach, da das Protoplasma selbst nicht zerlegt werden kann, die morphologische Einheit der lebenden Materie, in deren Raum sich dieselbe, sei es als zusammenhängende Masse, sei es durch Lücken unterbrochen ausbreitet; die Zelle ist der Elementarorganismus, der von verschiedener Grösse und verschiedenem Inhalt sein kann, aber als wesentliche Substanz das homogene, gleichartige, glasartig durchsichtige, zähflüssige Protoplasma enthält.

Gegenüber diesen herrschenden Anschauungen von der Gleichartigkeit des Protoplasmas giebt es eine noch älterc zweite Richtung von Bestrebungen, welche neben der anderen bisher nicht hat zur Geltung kommen können, und welche im Protoplasma noch eine weitere morphologische Zusammensetzung aus körperlichen Elementartheilen sucht, die dann selbst ihre lebendigen Fähigkeiten auf Grund einer molekularen Organisation entfalten mögen. Diese Bestrebungen drücken sich theils in Form von Wünschen und Vermuthungen, theils in Form von bestimmt geäusserten Anschauungen aus.

So sagt Brücke in seiner citirten Abhandlung: Ich nenne die Zellen Elementarorganismen, wie wir die Körper; welche bis jetzt chemisch nicht zerlegt worden sind, Iile- 
mente nemnen. So wenig die Unzerlegbarkcit dieser bewiesen ist, so wenig können wir dic Möglichlicit in Abrede stellen, dass nicht vielleicht die Zellen selbst noch wiederum aus andercn, noch klcineren Organismen zusammengeset\%t sind, welche zu ihnen in einem ahnlichen Verhältniss stehen, wic die Zellen zum Gesamntorganismus, aber wir habcn bis jetzt keinen Grund, diescs anzunelımen.

Aehnlich drickt sich Kölliker ${ }^{\mathrm{r}}$ ) aus, indem er sagt: Uvenn Bichat die Histologie durch die Aufstellung einer cinhcitlichen Grundlage und die scharfe Durchführung dersclbcn mehr im Allgemeinen begrindetc, so hat Schwann lurch seine Untersuchungen dieselbe im Einzelnen gesichert und sich so den zweiten Lorbecr in diesem Felde errungen. Was dic Wissenschaft seit Schwann bis auf unsere Targe noch leistete, war zwar von der grössten Bedeutung für die Physiologie und Medicin und zum Theil auch von rein wissenschaftlichem Standpunkte aus von hohem Werthe, allein alles dieses war doch nicht der Art, dass es um cinen namhaften Schritt weiter zu einem neuen Abschnitt ggefülırt hätte. Dieser Stand der Gewebelehre wird so lange dauern, als es nicht gelingt, um ein Wesentliches weiter in die Tiefe des Baues der lebenden Wesen zu schauen und auch die Elemente zu erfassen, aus denen das, was wir jetzt noch für einfach halten, zusammengesetzt ist. Solltc das aber je möglich werden, damn würde auch für die Histologie eine ncue Zeit bcgimnen, und dic Entdeckung des Gesctzes der Zellengenesc würde ebcnso oder noch mehr liedeutung gewinnen, als dic Lehre von der Zusammensctzung aller thierischen Gewebe aus Zellen.

Wir stellen dic Aeusscrungen dieser beiden Autoren hier voran, weil sie in cinfachster Weise den Standpunlit charakterisiren, auf welchem bis in unsere Tage die Lehre

1) Kölliker, Handbuch der Gewebelehre, 5. Aufl. 1867. S. 2. Gekürzles Citat. 
von den organisirten Formelementen gestanden hat. Es hat weder vor noch nach diesen Aeusserungen an Bemühungen gefehlt, der Frage von den wirklichen Elementarorganismen näher zu treten, aber alle diese Bemiihungen haben keinen Erfolg gehabt, weil sie mehr auf lypothetischen Anschauungen, als auf gefundenen Thatsachen beruhten.

Die Lehre von den Elementarorganismen ist in ihrer primitiven Form weit älter als die Zellenlehre selbst; es ist aber für den heutigen Biologen oft schwierig, sich in jene älteren Ideen hineinzudenken, und muss dieses jedenfalls mit Beriicksichtigung aller jener Unterschiede gcschehen, welche die Hilfsmittel der neueren Zeit vor denen der älteren auszeichnen. Darum thun wir vielleicht gut, folgende Worte Virchow's ${ }^{x}$ ) zu citiren, welcher, indem er selbst den Uebergang zur neueren Zeit mit erlebte und mit begründete, die Ansclıaungen jener älteren in folgender Weise schildert.

„Noch in den Elementa physiologiae von Haller findet man an die Spitze des ganzen Werkes, wo von den Elementen des Körpers gehandelt wird, die Faser gestellt. Haller braucht dabei den charakteristischen Ausdruck, dass dic Faser für den Physiologen das sei, was die Linie für den Geometer."

„Im Laufe des letzten Jahr\%elintes vom vorigen Jahrhundert begann indess schon eine gewisse Reaction gegen diese Faserlehre, und in der Schule der Naturphilosophen kam frühzeitig ein anderes Element zu Ehren, das aber in ciner viel mehr speculativen Weise begründet wurdc, nämlich das Kügelchen. Während dic Einen immer noch an der Faser festhielten, so glaubten Andere, wie in cler späteren Zeit noch Milne Edwards, so weit gehen zu dürfen, auch die Faser wieder aus linear aufgereihten Kügelchen zusammengesetzt zu denken. Diese Auffassung ist zum

1) Virchow, Die Cellularpathologie, 4. Aufl. 1871. S. 22 f. 
Theil hervorgegangen aus optischen Täuschungen bei der mikroskopischen Beobachtung. Die schlechte Methode, welche während des ganzen vorigen Jahrhunderts und eines Theiles des gegenwärtigen bestand, dass man mit mässigen Instrumenten in vollen Sonnenlicht beobachtete, brachte fast in allen mikroskopischen Objekten eine gewisse Dispersion des Lichtes, und der Beobachter bekam den Eindruck, als sïhe er weiter nichts, als Kügelchen. Andererseits cntsprach aber auch diese Anschauung den naturphilosophischen Vorstellungen von der ersten Entstehung alles Geformten."

„Diese Kügelchen (Körnchen, Granula, Molcküle) haben sich sonderbarer Weise bis in die moderne Histologie hinein crhalten, und es gab bis vor Kurzem wenige histologische Werke, welche nicht mit den Elementarkörnchen anfingen. Hier und da sind noch vor nicht langer Zeit diese Ansichten von der Kugelnatur der Elementartheile so überwiegend gewesen, dass auf sie die Zusammensetzung, sowohl der ersten Gewebe im Embryo, als auch der späteren begrindet wurde. Man dachte sich, dass eine Zelle in der Weise entstände, dass die Kügelchen sich sphärisch zur Membran ordneten, innerhalb deren sich andere Kügelchen als Inhalt erhielten. Noch von Baumgärtner und Arnold ist in diesen Sinne gegen die Zellentheorie gekämpft worden." „In einer gewissen Weise hat diese Auffassung in der lintwickelungsgeschichte cine Stütze gcfunden, in der sogenannten Umhüllungstheorie (Henle). Danach dachte man sich, dass, während ursprünglich eine Menge von Elementarkügelchen zerstreut vorhanden wären, diese sich unter bestimmten Verhältnissen zusammenlagerten, nicht in Form sphärischer Membranen, sondern zu einem compakten Haufen, einer Kugel (Klümpchen), und dass diese Kugel der Ausgangspunkt der weiteren Bildung werde, indem durch Differenzirung der Masse, durch Apposition oder Intussusception aussen eine Membran, innen ein Kern entstehe." 
„Gegenwärtig kann man weder die Faser, noch das Kugclehen oder Elementarkörnchen als cinen histologischen Ausgangspunkt betrachten."

Diese älteren Anschauungen nun, wie sie hicr von Virchow so trefflich wiedergegeben werden, sind von cinzclncn Autoren bis in die ncueste Zcit hinein mit grosscm Eiffer verfochten worden, insbesondere von Béchamp und Estor. Beide Autoren, indem sie meist gemeinschaftlich ihrc Anschauungen äusserten, stehen ganz auf dem Boden der alten Umhïllungstheorie. Auch nach ihnen soll dic Zelle cntstehen, indem die Elementarkörnchen, welche sic Mikrozymas nennen, sich zusammenlegen und durch Differenzirung ihrer Masse sich zu Zellen umbilden. Henle mit sciner Umhüllungstheorie gilt ihnen daher als dicjenige Autorität, an deren Aeusserungen sic vorzugsweise gernc anknuipfen, und um so lieber, als sie selbst, wie es schcint, nicht Morphologen sind. Neu ist bei ihnen noch die zwcitc Idce, welche vorzugswcise ihr persönliches Interesse in Anspruch nimmt, dass dieselben Kügelchen durch Zerfall der Zelle wieder frei werden können und so Bacterien bildon.

Alle ernsten Bemühungen unserer Zeit haben aber in bciden Fällen zum entgegengesetzten Resultat geführt. Der Lehrsatz Virchow's, omnis ccllula e cellula, welcher der Unhüllungstheoric gegcnübersteht, ist heute mehr denn jc anerkannt, nicht auf Grund von Hypothesen, sondern auf Grund jener Thatsachen, wie sie insbesondere durch dic Erscheinungen der Karyokinese sicher gestellt worden sind, und die Integrität der Abstammung der Spaltpilze, wic sic von den Versuchen Pasteur's ihren wesentlichen Ausgang genommen hat, ist bis jetzt durch die weitercn Beobachtungen immer mehr begründet, nicht negirt worden; auch dic nicht minder verfehlten Bemuihungen Wiegand's ${ }^{\mathrm{I}}$ ), wclcher von seinem Standpunkte als Botaniker ebcnfalls

1) A. Wiegand, Entstehung und Fermentwirkung der Bacterien, Marbury 1881. Derselbe, Das l'rotoplasma als Fermentorganismus. Marburg 1888. 
cine Anamorphose des l'rotoplasmas zu Bacterien behauptet, haben hieran nichts zu ändern vermocht. Dic Opposition gegen Virchow und l'asteur ist aber überall dasjenige Moment. welches in den Auslassungen jencr beiden Autoren insbesondere hervortritt. Diese Opposition hätte trotz ihres verfehlten Charakters ihren Nutzen gehabt, wenn es jenen Autoren gelungen wäre, die Elemente der Zelle zu sehen und zu demonstriren. Sic haben aber nicht mehr, vielleicht weniger gesehen, als die anderen Mikroskopiker vor ihnen auch. Es bleibt daher an ihnen nichts anderes anzuerkcnnen übrig, als die Begcisterung, mit welcher sie die alten Ilcen von den Elementarkörnchen verfochten haben. ${ }^{\mathrm{x}}$ )

Trotzdem scheint es, als wenn die alte Lchre von den Elementarkörnchen ihre lierechtigung hat. 1)ic Zellen sind nicht Elementarorganismen, sondern Colonieen von solchen mit eigenartigen Gesetzen der Colonisation ${ }^{2}$; die Zellen entstehen aber nicht durch das Zusammentreten der Kügelchen, sondern sie sind daraus in jenen geschichtlichen Perioden entstanden, dic den mikroskopischen Elementen gerade so eigen sind, wie den groben Formen der Lebewesen auch; die Elementarkörnchen der Zellen, welche noch heutc ihre analogen

I) Vergl, hieriiber die zahlreichen Abhandlungen, welche in den Comptes rendues seit etwa 1860 bis heute ersehienen sind. Ausserdcm 1. Béchamp, Les microzymas. Paris 1883 und A. Estor, lo la constitution élémentaire tles tissus. Montpellier 1882.

Lim die Mangelhaftigkeit der Peobachtungen jener Autoren \%u priffen, lrancht man nur die Abbildungen in dem citirten Werke Búchan p's, die cinzigen ïbrigens, welche jene Autoren gelicfert haben, zu betrachten; es erselheint dann klar, dass von vielen anderen Autoren älterer und nencrer \%cit sowohl an der thierischen, wie auch an der Pflanzenzelle bessere und ausgriebigere Beobachtungen gemacht wordcn sind.

Bci der L'nfruchtbarkeit ihrer Opposition gegen l'astcur und Virchow und bei der Mangelhaftigleit ihrer thatsäehlichen Befunde nimmt es daher nicht Wunder, wenn, wie Estor sich bitter beklagt (I. .. S.VIII), sellost die Mitglieder des französischen Instituts ihnen in ihrem eigenen Intercsse abgerathen haben, weiter auf dem betretenen Wege vorzugehen.

2) Vergl. Lie Cienese der \%elle. Festschrift für Carl Ludwig 1887. 
Vertreter in den Mikroorganismen haben und welche seit jenen Perioden in den Zellen existiren, vermögen nicht mehr selbstständige Lebewesen $\%$ u werden.

Beide Richtungen nun, sowohl diejenige, welche die Gleichartigkeit des Protoplasmas betont, als auch diejenige, welche die Elementarkörnchen als die Grundelemente der lebenden Materie betrachtet, haben in der Art, wic sic bisher vertreten worden sind, ihre Fehler aufzuwcisen. Im crsten Falle leugnete man Dinge, weil man sie nicht sah, im anderen behauptete man Dinge, obwohl man sie nicht sah; zu Beidem hatte man kein Recht.

Jene Anschauung von der Gleichartigkeit des Protoplasmas stützt sich zum grössten Theil auf Beobachtungen, welche an bestimmten lebenden Objekten angestellt seincr Zeit grundlegend für die Betrachtung des Protoplasmas als Ganzes waren, niemals aber für die analytische Bctrachtung desselben massgebend sein und bleiben durften. Die sich bewegenden Plasmaströme der Pflanzenzellen, die Bewegungserscheinungen an den Rhizopoden, Myxomyceten, die lebenden Leucocyten des Blutes waren cs, von welchen her allgemeine Folgerungen über den Bau des Protoplasmas hergelcitet wurden und besonders von Seiten der Botaniker noch heute hergeleitet werden.

Die lcbenden Objelite haben für den Beobachter gcwiss etwas ausserordentlich Fesselndes, und Niemand wird den Werth solcher Beobachtungen leugnen, odcr nur herabzusetzen suchen; will man jedoch den Bau des Protoplasmas sehen, so findet man in ihnen nur selten einen sicheren Anhalt. Man sieht eben, wic dieses v. Mohl, Schultzc, Kühner), Lieberkühn²) und viele Anderc in oft klassischer Wcise beschrieben haben, das schöne Spiel der in und mit der hellen Grundsubstanz strömenden

1) IV. Kii hne, Untersuchungen über das Protoplasma. Leipzig 1864.

2) N. Lieberkühn, Ueber Bewegungserscheinungen der Zellen. Marburg 18;0. 
Körnchen; man sieht oft die peripheren Theile frei von diesen; bald ist es Vergrösserung, bald Verkleinerung der einzelnen Theile, bald Trennung, bald Verschmelzung derselben, welche uns entgegentreten, und vicles Geistvolle ist darüber zu sagen und gesagt worden. Warum aber diese selben Objckte, welche nach der einen Seitc hin so wunderbare Schönhciten offenbaren, auch anderweitig massgebend sein sollen, das ist nicht einzusehen.

Es scheint, als wenn für das Studium des protoplasmatischen Baues zwei Grundsütze massgebend sein müsscn: dic Anwendung der künstlichen Methoden, welche uns weitcr in die Tiefe jenes Baues hineinzuführen vermögen, als die natiirlichen Beobachtungen und die Wahl geeigneter Objcktc, deren Elemente sich durch ihre Deutlichkeit auszcichnen. Wenn man aus unpassenden Objekten mit unpassenden Methoden allgemeine Folgerungen herleiten will, so weiss man eben nicht, was feinere mikroskopische Analyse bedeutet; es ist hier einc der alltäglichsten Erfahrungen, dass Dinge, welche vorhanden sind, wegen ihrer Kleinheit oder aus anderen Gründen nicht gesehen werden, und je weiter die Erfahrungen in der feineren mikroskopischen Analyse rcichen, desto mehr kommt man zu der Ansicht, dass das, was wir von den morphologischen Elementen schen, nur ein Bruchtheil ist von dem, was wir nicht schen. Der Mikrologe ist selten in der Lage, gegenüber diescn noch nicht gesehenen Dingen mit vorgefasstem Willen cinen Erfolg zu erreichen; seinc Kunst besteht darin, den Dingen geduldig nachzugehen und ihnen ihre Eigenlieiten abzulauschen, wo und wic er sic erreichen kann; wer hier an Andere unberechtigte Forderungen macht, der stellt sich auf den Standpunkt desjenigen, der nicht gelernt hat, sein eigenes Können und das der Andcren abzuwägen.

Die lcbenden Objekte haben zunächst den grossen Nachtheil, dass die Sichtbarkeit der Elemente von mancherlei Zufälligkeiten abhängt; cs bedarf nur eines annähernden 
Ausgleiches der Brechungsunterschiede, um selbst solche Elemente unsichtbar zu machen, die wegen ihrer Grösse sonst bequem der Beobachtung zugänglich wären. Die küinstlichen Methoden sind von solchen Zufälligkeiten in hohem Grade unablängig, und es liegt nur in unscrem Können, wie intensiv wir die Differenzen der Sichtbarkcit erzeugen. Da die Grösse der hier in Betracht kommenden Elemente oft unterhalb und oft an der Leistungsgrenzc der Mikroskope liegt. so müssen wir un so mehr bemüht scin, dic Kräfte derselben bis zum Extrem auszunützcn; das können wir aber, wie dem Einsichtigen leicht klar sein wird, an den natürlichen Objekten nicht durchfuihren.

Sowohl für die natürliche als auch für dic künstliche Bearbeitung jedoch werden wir nicht beliebige Objekte wählen, sondern diejenigen bevorzugen, wo die Grösse und Art der Elemente die Beobachtung erleichtert, und je leichter und sicherer dicse Beobachtung ist, desto willkommener muss uns ein solches Objekt sein. Unter den vielen Objektcn zcichnen sich die echten Pigmentzellen dadurch aus, dass sic bereits olne Kunsteingriffe beobachtet werden können; wcnn sie uns so direkt einen Einblick in ihr Inneres gestatten. so miissen sie uns massgebender sein, als alle farblosen Zcllen, die dieses nicht thun. Wenn die Muskelfaser uns bei geringer Mühewaltung den Bau des Protoplasmas in grosscr Vollständigkcit darbietet, so wird sie uns das l'rototyp des protoplasmatischen Baues sein und nicht die Sarkode, an welcher wir nichts schen; wir werden, wenn es uns gelingt, in anderen Zellen analoge Verhältnisse aufzudecken, dann mehr Recht haben aus den Pigmentzellen und Muskelfasern allgemcinere Folgerungen zu ziehen, als diejenigen, welche diescs von der Sarkode her gethan habcn, denn positive Beobachtungen beweisen, nicht negative. Wer dann ein Interesse daran hat zu wissen, ob die Sarkode einc Structur hat oder nicht, der mag sich doch darum bemühen; will er alsdann behaupten, dass sie structurlos sei, dann hat cr es zu boweisen, nicht ein Anderer; ohne 
diesen Beweis abcr allgemeine Folgerungen zu zichen, ist gewiss verfehlt. Wenn die liotaniker, welche weder Pigmentzellen noch Muskclfasern haben, bei der alten Mohl'schen Definition noch bis heute stehen geblieben sind, so ist das nicht zu verwundern; den Zootomen aber müssten jene günstigen Objekte doch wohl der Ausgangspunkt sein, von welchem aus er sich bemuihen konnte weiter zu kommen, statt einfach den Inhalt der Muskelfasern auf eine Ablagerung der quergestreiften Elemente, und den der Pigmentzellen auf eine Absetzung von neuen Stoffen in unlöslicher Form zurückzuführen (Kölliker ${ }^{\mathrm{T}}$ ). Sehen wir von denjenigen Fällen ab, wo es sich um regellose, resp. krystallinische Niederschläge pigmentirter Stoffe in den Zellen handelt, so sind mancherlei Gründe vorhanden, sowohl dic Körnchen der echten Pigmentzellen als auch die Elemente der Muskelfasern für organisirte Gebilde zu halten; organisirte Gebilde aber entstehen, soweit unsere Kenntnisse von den natürlichen Dingen reichen, nicht durch Ablagerung oder Absetzung. Es liegt hier nahe anzunehmen, dass die von der Natur gefärbten echten Pigmentkörnchen den durch Kunst färbbaren Granulis der andern Zellen analog sind, wenigstens hat mich diese Annahme seiner Zeit dazu gefülırt, solche künstliche Färbungen zu suchen, welche eincn Ersatz für die natürlichen Färbungen der Pigmentzellen bilden sollten.

Auch von Seiten der Botaniker hat es nicht völlig an Bemühungen gefehlt, dem Protoplasma mit künstlichen Methoden näher zu treten. Schmitz ${ }^{2}$ ) giebt an, bei Picrinpräparaten mit Haematoxylin gefärbte Punktirungen des Cytoplasmas erhalten zu haben; die Ungunst der Pflanzenobjekte für künstliche Bearbeitung scheint ihn jedoch abgehalten zu haben, hierin weiter vorzugehen.

1) Kölliker, Handbuch der Gewebelchre. 6. Auf. 1889. S. 31 .

2) F. Schmitz, Untersuchungen uiber die Structur des Protoplasmas und der \%ellkerne der l'fanzenzellen. Sitzungsherichte der niederrheinischen Gesellschaft \%u Bonn 1880. 
So sehr auch die Pflanzenzelle für die Beobachtung vieler lebenden Vorgänge geeignet ist, ihr eigentliches l'rotoplasma ist um so schwieriger zu erreichen; die Neigung desselben zur Bildung von grossen Vacuolen ist so vorherrschend, dass man, um das Cy'toplasma besonders an den für künstliche liearbeitung nothwendigen dünnen Schnitten erfolgreich untersuchen zu können, auf wenige Jugendformen angewiesen ist; hierzu kommt noch das häufige Vorhandensein von Chlorophyllkörnern, Leucoplasten etc., welche das spärliche Cytoplasma verdecken. Ich habe es in Gemeinschaft mit einem Botaniker versucht, die an der thierischen Zelle erprobten Methoden auf die Pflanzenzelle zu übertragen; hierbei hat sich jedoch die Ungunst der Letzteren so evident herausgestellt, dass wohl Analogieen zur thierischen Zelle nachweisbar waren, eine wesentliche Förderung der Granulafrage von der Pflanzenzelle aber schwer zu erwarten ist. ${ }^{\mathrm{r}}$ ) Man braucht nur die von Oskar Schultze ${ }^{2}$ ) an Thieren angestellten Beobachtungen über die vitale Metylenblaureaction der Zellgranula mit den ärmlichen Bildchen und spärlichen Erscheinungen zu vergleichen, welche auf ähnliche Weise gelegentlich an der Pflanzenzelle gewonnen worden sind, um jenen Unterschied genügend zu übersehen. Die Beobachtungen an den Pflanzenzellen werden in vielfacher Weise für das Studium der lebenden Vorgänge massgebend bleiben, aber jenes weitere Eindringen in den protoplasmatischen $\mathrm{Bau}$, wie es vermittelst der künstlichen Methoden erreicht werden kann, werden sie kaum gestatten; hierzu eignen sich die thierischen Zellen augenscheinlich in weit höherem Grade. Es dürfte zweckmässig, ja für einen weiteren Fortschritt nothwendig sein, dass

I) Herr Dr. A. Zimmcrmann, Docent der Botanik in Tübingen, hat vor einiger Zeit ein paar Monate bei mir mit den Granulamethoden gearbeitet; er gedachte seine Untersuchungen, die in Bezug auf Specialfragen der Botanik vieles Interessante boten, in Tübingen fortzusetzen und seiner \%eit zu veröffentlichen.

2) O. Schultze, Die vitale Metylenblaurenction der Zellgranula. Allat. Anzeiger $\mathbf{1} 887$. 
sich die Bestrebungen auf diesen beiden Gebicten in harmonischer Weise ergänzen.

An der thierischen 'Lelle sind auch früher schon an vereinzelten Objekten mit kiinstlichen Methoden günstige Resultate erzielt worden. So hat Ehrlich die gröberen Granulationen verschiedener Leucocyten gefärbt, van Beneden spricht von corps baccilliformes, welche er gelegentlich in Zellen gesehen hat, Kupffer hat im Axencylinder, wie ich es nach meinen jetzigen Erfahrungen auffassen möchte, fibrillär angeordnete Granula durch Färbung demonstrirt; dennoch sind diese Beobachtungen sowohl von diesen Autoren selbst, als auch von Andern nur als Specialitäten und veremzelte Erscheinungen aufgefasst worden. Seit dem Bekanntwerden meiner Granulauntersuchungen ') haben sich die Angaben über das Sichtbarsein von Körnerelementen in den Zellen bereits erheblich vermehrt, und man scheint sich bereits daran zu gewöhnen darauf zu achten, wo sie gelegentlich auch ungefärbt oder als gefärbte Nebenprodukte der Beobachtung erkennbar werden, ja Manche halten es heute schon für selbstverständlich, dass die Zelle kein Elementarorganismus ist. Es lässt sich hoffen, dass, wenn erst die für die Untersuchung der Granula geeigneten Methoden in Aller Händen sind ${ }^{2}$ ), dieses Gebiet der Biologie bald durch rüstige Mitarbeiter gefördert werden wird. Das Endziel unserer Bestrebungen aber soll sein den Satz immer mehr wahrscheinlich zu machen: es giebt keine gleichartige Sarkode, es gicbt nur ein polymeres Protoplasma.

Von den allgemeineren Bemühungen, das Princip im Bau des l'rotoplasmas zu finden, kann man, abgesehen von

I) Studien iiber die Zelle. Leiprig 1886. - Die Genese der \%elle. Festsehrift für Carl Ludwig 1887. - Die Struetur des Zellkerns. Arcl. f. Anat. u. Phys. 1 889. - Ueber dic Fettumsetzungen im Organismus. Ebendia. Eine grössere Zahl von Granulabildern wurden auf den Anatomen-Versammlungen zu Leipzig und Würzburg demonstrirt.

2) Die Metloden finden sieh beschrieben in einer im Druck befindlichen Arbeit: Die Elementarorganismen und ihre liesiehungen z:11 den \%ellen. 
den schon oft gesehenen und beschriebenen Fascr- und Fibrillenbildungen, welche, wie oben erwähnt, Kölliker für wichtige Einzelheiten des protoplasmatischen Baues erklärt, und auf deren Bedeutung wir an einem anderen Orte bereits näher eingegangen sind ${ }^{\mathrm{x}}$ ) und später noch des Weiteren eingehen werden, noch die Anschauung von der primären Netzstructur des Protoplasmas hervorheben, wie sie insbesondere von Heitzmann ${ }^{2}$ ) an thierischen Zellen und von Frommann3) an Pflanzenzellen beobachtet worden ist.

Die Bemühungen beider Autoren bezeichnen insofern schon einen Fortschritt, als von ihrer Seite bereits einc strengere Auswahl der für Structurstudien geeigneten $\mathrm{Ob}$ jekte stattgefunden hat; indem sie eifrig danach suchten, wo etwa sichtbarliche Formerscheinungen im Protoplasma zu entdecken waren, setzten sie daselbst alle Mühe heran. Während die älteren Autoren durch ihre klassische Beobachtungsgabe das Wesen des Protoplasmas als Ganzes in vielen Punkten klar gelegt haben, finden sich in den Bemühungen von Heitzmann und Frommann die ersten Anfänge dafür, die Elemente zu demonstriren, aus denen sich dasselbe zusammensetzt. Beide kamen sie zu dem Resultat, dass die Substanz des Protoplasmas in äusserst feinen Netzen angeordnet sei, dessen Knotenpunkte den Eindruck von Körnchen machen; hierin sollte das Wesen des protoplasmatischen Baues bestehen.

Was es mit diesen Netzen meist für eine Bewandtniss luat, dafür möchte ich nur ein Beispiel anführen. Frommann fand in den Staubfadenhaaren von Tradescantia ein ausgezeichnetes Objekt, um in den Kernen der dort vor-

1) Vergl. Die Genese der Zelle.

2) C. Heitzmann, Untersuehungen über das Protoplasma. Wiener Sitzungsberichte 1873. Mikroskopische L'ntersuchungen des Thicrkörpers. Wien 1883.

3) C. Frommann, Beohaehtungen üher Struetur und Bewegungserscheinungen des Protoplasma der P'fanzenzellen. Jena 1880. 
handenen 'Zellen ein ausserordentlich regelnässiges feinmaschiges Netzwerk lebend $z u$ demonstriren. Wenn ich dasselbe Objelit ebenfalls in frischem Zustande untersuche, so finde ich, dass dasselbe ausgezeichnet ist, un die Granulastructur des Kemes zu demonstriren; das heisst Frommann halt die lntergranulasubstanz für das positive Bild, während er die Granula für Lücken ansieht, während ich die Lücken für positive Granula halte, das Netzwerk aber fuir intergranulär.

Jedenfalls ist dieses Beispiel charakteristisch dafiir, dass gleiche Beobachtungen an lebenden Objekten, deren Sichtbarkeit fast immer nur auf Brechungsdifferenzen beruht, leicht zu entgegengesetzten Folgerungen führen können, besonders da, wo es sich um die feinsten Formelemente handelt. Die Entscheidung kann naturgemäss nur durch künstliche Hilfsmittel gebracht Werden; wenn es dadurch gelingt, an Stelle der Lücken des Netzwerkes positive Körper mit specifischer Färbungsreaction nachzuweisen, so ist die Structur granulär, das Netzwerk aber intergranulär. Damit soll nicht gesagt sein, dass nicht auch dem intergranulären Netzwerke noch eine vielleicht viel feinere $\mathrm{Zu-}$ sammensetzung aus Elementarkörperchen zukonmt; ja es ist mir dieses nicht unwahrscheinlich, wie ich es bereits in meiner Mittheilung über die Structur des Zellkernes erwähnt habe.

Lebende Objekte geben also nicht nur selten einen sicheren Anhalt für die Beobachtung der Structur des Protoplasmas, sondern sie führen auch da, wo sie dieses thun, leicht zu Tauschungen. Da weder Heitzmann noch Frommann küinstliche Methoden angewendet haben, so ist es auch ihnen nicht gelungen, das Princip im Bau des Protoplasmas aufzudeclien, obwohl ihre Beobachtungen zu den besten gehören, welche über die Structur desselben angestellt worden sind.

Es scheint daher für das Studium des Protoplasmas der richtige $W c g$ zu sein, vorzugsweise mit Hilfe der zu- 
verlassigeren und weiter eindringenden künstlichen Methoden, und im Anschluss an so prägnante Objekte, wie sie die P'igmentzellen und die Muskelfasem des thierischen Organismus darbieten: analoge Verhältnisse auch in anderen Zellen zu suchen; finden wir solche Analogieen, so werden wir mehr Recht haben, allgemeine Folgerungen daraus zu ziehen, als diejenigen, welche ihre Anschauungen von der Gleichartigkeit des Protoplasmas auf die negativen Befunde an der Sarliode begründen.

Haben die Vertreter dieser Anschauungen Recht, dann lat die Morphologie bereits ihre Grenze erreicht, und es bleibt nur die Lehre von der molekularen Organisation übrig, welche für grübelnde Leute gewiss viel Reizvolles hat, aber doch selbst erst der richtigen morphologischen Unterlagen bedarf, um eine Berechtigung ihrer Existenz $z u$ besitzen.

Noch haben wir diese Grenze der Morphologie nicht crreicht. Mag jener genetische Plan, wie wir ihn oben in wenigen Worten zusammengedrängt haben, auch ein Unbekanntes sein, das bewiesen werden muss, vielleicht kann er uns doch den Weg zeigen, wie wir zu einem Verständniss des Bekannten und Erreichbaren gelangen. Wenn wir Schritt für Schritt durch immer feinere Methoden das Gebiet des Sichtbaren erweitern, so gelingt es vielleicht doch allmählich, Vieles von dem zu sehen, was scheinbar nicht vorhanden ist; das was in dieser Beziehung schon erreicht wurde, lässt die Hoffnung auf weitere Fortschritte als möglich erscheinen. Es mag hierin vielleicht eine schwerc Aufgabe liegen, aber es lohnt der Mühe wohl, hier seine Kräfte heranzusetzen und so unserem Wissen eine neue WVelt zu erobern. - 